\title{
Whole grain for whom and why?
}

\author{
Wenche Frølich ${ }^{1}$ and Per Aman ${ }^{2}$ \\ 'Norwegian School of Hotel Management, University of Stavanger, Jar, Norway; ${ }^{2}$ Department of Food Science, \\ Swedish University of Agricultural Sciences (SLU), Uppsala, Sweden
}

Abstract

A definition of whole grain is a critical first step in investigating health claims for whole grain and its products. Today, there is no internationally accepted definition of whole grain. Some existing definitions are broad and commodity-based, including grains with similar end uses, while others are more restricted. Scientific knowledge must be the basis for inclusion of certain grains. It is better to start with a restricted list of grains (a precautionary principle) and extend this as more knowledge becomes available. An exact definition of the raw materials (milled, cracked, crushed, rolled, or flaked) and knowledge of the components providing health effects would appear to be crucial issues for the European authorities when approving health claims. It is important that health claims are evidence-based, sustainable, and officially validated.

Keywords: whole grain; definition; raw materials; health claims

Received: 16 December 2009; Revised: 2 February 20 I0; Accepted: 3 February 20I0; Published: 12 March 2010

$\mathrm{F}$ or a long time there has been a strong international trend to market whole grain foods as healthy. In many cases it has been difficult for consumers to obtain reliable information about food items and their health effects, and thus select the most desirable products from a nutritional point of view. Consumers, at least in Scandinavia, are well aware that whole grain is healthy, but are less well informed about what whole grain is and its relationships to health. Several population studies have shown correlations between increased intake of whole grain and decreased risk of developing diet-related diseases, such as cardiovascular disease (CVD), diabetes, certain cancers, and obesity $(1,2)$. However, these studies have mainly been conducted in Western populations and have included different definitions of whole grain. In Western populations, whole grain intake typically comes from wheat, oats, rye, barley, and sometimes popcorn, and therefore the estimated risk reductions are most likely associated with intake of these cereals. Results from intervention studies have been more variable. Some studies have shown no effects of whole grain consumption on intermediate endpoints for CVD and diabetes, while others have shown effects (3). A number of these intervention studies have evaluated the effects of whole grain from oats, wheat, rye, and barley. In order to provide consumers with appropriate dietary advice and to give the industry the opportunity to develop and market innovative and healthy foods, a definition of whole grain based on compelling evidence from a totality of population and intervention studies is needed.

\section{Cereals: a good source for nutrients}

Whole grain cereals are well known as a major source of dietary carbohydrate and protein, as well as containing high amounts of a variety of dietary fibers and co-passengers, i.e. minerals, vitamins, and other bioactive components. These latter components are mainly present in the outer parts of the grain or in the germ, and are removed with the bran during extraction of sifted flour. As a result, health authorities around the world are recommending increased intake of whole grain cereals. However, evidence-based recommendations on the exact amounts and types of whole grains to be consumed are rare.

Consumers are well aware that whole grain is healthy and that consumption of whole grain foods should be increased. However, there is a mismatch between whole grain recommendations and the actual behavior of consumers. Knowledge among consumers of the nutritional content and health effects of grain seems to be limited. Questions that ought to be raised in this context are therefore: Do consumers know what is meant by whole grain? Do they know which grains are included in this group? Are all whole grains or whole grain foods the same when it comes to nutritional content and health effects? 


\section{Whole grain definitions}

With the current interest in whole grain, not only among health authorities and consumers, but also within the food industry, a definition of whole grain is urgently needed. Most of the target groups - scientists, industries, authorities, and consumers - are interested in the health benefits in whole grain. Even depending on different aims (e.g. dietary recommendation, nutrition claims, or health claims), the definition of whole grain needs to be strict. A generally higher content of vitamins, minerals, and dietary fiber is not enough for dietary recommendations on whole grain even if this might be enough regarding nutrient content behind nutritional claims for selected nutrients. Knowledge about the exact content of nutrients in whole grains (milled, cracked, crushed, rolled, or flaked) of different cereals is, however, needed, both for dietary recommendation and nutrient claims. Only those whole grains, which have a similar content of nutrients should be included. To demonstrate the beneficial effects and/or disease risk reduction for whole grain, an exact and strict definition must be available.

To date, however, there has been no internationally accepted definition of whole grain. Currently, the scientific community is actively involved in discussions regarding an official definition, including issues such as the types and parts of grains that should be included in the definition. An important aspect in finding an applicable definition is the target group: individual consumers, to facilitate their choice of healthy food alternatives; the scientific community, to establish a uniform dietary factor to study in relation to health; the authorities, to allow them to make recommendations; or the food industry, to produce new whole grain products. A definition of whole grain would almost certainly benefit all these interests. However, for a definition to be useful in a long-term perspective, it must be grounded on evidence-based nutritional aspects rather than simply classification of food items.

In the scientific literature and popular press, much of the discussion concerns the seeds that should be included in the definition 'whole grain' (4). It is not clear whether such a definition should be based solely on whole grain cereals or whether the so-called pseudocereals and other starch-rich seeds should also be included, and the rationale behind their inclusion has to be defined.

Cereals are members of the grass family (Poaceae or Gramineae) and produce dry one-seeded fruits (caryopsis) which are commonly called a kernel or grain (5). All cereals consist of a fruit coat (pericarp) surrounding the seed. The seed contains an embryo (germ) and an endosperm surrounded by a nucellar epidermis and a seed coat (testa). In general, all cereals have broadly similar proportions of these botanical structures. However, it is not possible to specify a standard ratio for the different structures, since these vary within and between cereals. In addition, some cereals, such as rice, oats, and barley retain their husk during threshing and this must be removed to produce acceptable foods for humans. Bran is a technical fraction from the milling industry. It generally comprises the fruit wall, seed wall, aleurone layer, and small amounts of the starchy endosperm and germ. The composition of a bran fraction is highly dependent on the milling technology and type of grain used.

Pseudocereals, such as amaranth, quinoa, and buckwheat are not members of the grass family, but because of the high starch content in their seeds and their use in cereal-like products, it has been suggested that they be classified as whole grain together with the cereals in the grass family. These seeds contain no gluten and are therefore suitable alternatives for people with celiac disease.

Pulses or grain legumes are other seeds with a high content of starch that can be used in different types of cereal-like products. Because of these characteristics, they may fall into the definition of whole grain although they are generally not regarded as such.

\section{Existing definitions of whole grain}

\section{American Association of Cereal Chemists (AACC) International and American Whole Grain Council (WGC) definition}

Whole grain was defined back in 1999 by American Association of Cereal Chemists (AACC) International (6): 'Whole grain shall consist of the intact, ground, cracked or flaked caryopsis whose principal anatomical components - the starchy endosperm, germ and bran are present in the same relative proportion as they exist in the intact caryopsis.' Inclusion or noninclusion in this definition is not based on fiber content, and although nuts and legumes are regarded as healthy plant foods, they are not included in the definition. Pseudocereals (buckwheat, amaranth, and quinoa), on the other hand, are included since they are considered to have similar macronutrient composition to whole grain cereals and are eaten in the same way.

The AACC International definition of whole grain was adopted by the US Food and Drug Administration (FDA) in the document 'Whole Grain Label Statements' in 2006, to provide guidance for the industry (7).

The American Whole Grain Council (WGC) defines whole grain in a similar way to AACC International, using the following wording in their definition in 2004 (8): 'Whole grains or foods made from them contain all the essential parts and naturally occurring nutrients of the entire grain seed. If the grain has been processed (e.g. cracked, crushed, rolled, extruded, and/or cooked), the food product should deliver approximately the same rich 
balance of nutrients that are found in the original grain seed.'

This definition includes the following cereals and pseudocereals (and forms of these): amaranth, barley, buckwheat, corn, including whole cornmeal and popcorn, millet, oats, including oatmeal, quinoa, rice, both brown rice and colored rice, rye, sorghum (also called milo), teff, triticale, wheat, including varieties such as spelt, emmer, farro, einkorn, $\operatorname{Kamut}^{\circledR}$, durum and forms, such as bulgur, cracked wheat and wheatberries, and wild rice. It also includes more unusual cereals belonging to the grass family such as canary seed, Job's tears, montina, and fonio when consumed with all of their bran, germ, and endosperm.

Oilseeds and legumes (such as flax, chia, sunflower seeds, soy, chickpeas, etc.) are not considered whole grains by the WGC, AACC International, or the FDA.

\section{Definition in Denmark, Sweden, and the Scandinavian keyhole}

A Danish Task Force (2) from 2008 defines whole grain as intact, ground, cracked, or flaked kernels after removal of the husks. In this definition the nine main cereals within the grass family (barley, oats, wheat, rye, rice, millet, maize, sorghum, and triticale) are included. It is permissible to combine different milling fractions, but the relative proportions of bran, starchy endosperm, and germ must be the same as in the intact kernels. Only dry flour of whole maize is included, but not fresh maize and popcorn. Pseudocereals are not included.

In Sweden, the National Food Administration uses a similar definition of whole grain to that of the Danish Task Force, but does not include triticale, since this cereal is not used in Swedish human foods.

The three Scandinavian countries, Denmark, Sweden, and Norway, have agreed on common rules for declaration of healthy foods in a system entitled 'The Scandinavian Keyhole' (9). These rules, accepted by the health authorities in the three countries, include a definition of whole grain. 'Whole grain is defined as intact and processed (dehulled, ground, cracked, flaked, or the like) products where endosperm, germ, and bran are present in the same proportions as in the intact grain. If these fractions are separated under processing, they should be added back so that the final product has approximately the same relative proportions of the three parts as in the intact grain. The whole grain definition includes the following whole grain cereals: wheat, rye, oats, barley, maize (dry seeds), rice, millet, and sorghum. Wild rice, quinoa, amaranth, and buckwheat are not included.'

\section{Health claims in the USA and Europe}

A whole grain health claim was the first to be allowed by the FDA in 1999: 'Diet rich in whole grain foods and other plant foods and low in fat, saturated fat and cholesterol may reduce the risk of heart disease and some cancers' (10). The product should contain 51\% whole grain cereals or more per reference amount. This is easily achieved for dry foods such as breakfast cereals, but not for products with higher moisture contents such as bread. The FDA has produced a draft guidance to industry about what they consider to be whole grain and to assist the manufacturers in labeling their products (11). Three health claims have also been approved for grain products (not for whole grains) relating to coronary heart disease and certain cancers $(3,12)$.

A health claim was subsequently accepted by the Joint Health Claim Initiative in UK in 2002: 'People with a healthy heart tend to eat more whole grain foods as part of a healthy lifestyle' (13). The food should contain 51\% or more whole grain ingredients by weight per serving. The term 'whole grain' refers to the major cereal grains, such as wheat, rice, maize, and oats.

A Swedish Code of practice entitled 'Health Claims in the Labeling and Marketing of Food Products: The Food industry's Rules' (14) has been developed in close co-operation with relevant authorities. In 2003, a whole grain claim was adopted: 'A healthy lifestyle and a balanced diet rich in whole grain products reduce the risk of heart disease (http://www. snf.ideon.se). Product X is a good source of whole grain.' The food should contain at least $50 \%$ whole grain from wheat, rye, oats, or barley on a dry matter basis.

Awaiting European Union regulations, health claims in Europe primarily have been country-specific. Scientific substantiation of claims is one of the most important aspects of providing truthful information to consumers, satisfying regulatory requirements, and allowing fair market competition.

\section{Basis for health claims for grains}

Currently, accepted health claims for grains have mostly been substantiated by observational/epidemiological studies. Unfortunately, different definitions for whole grain have been used. While AACC International has used the broadest inclusion criteria for grains (both cereals and pseudocereals), the Swedish and UK definitions have included only the most commonly eaten grains. Another problem may arise in that some studies include not only whole grain, but also products containing extra added bran and germ.

In population studies no cause and effect of the whole grain can be demonstrated. Assessment of intake is usually based on self-reporting, which is prone to systematic and random measurement errors (15). Furthermore, the lack of a general definition for whole grain foods also raises questions about the accuracy of reporting about the amount of wholegrain products consumed by the study participants. 
There have only been a few intervention studies that relate whole grain intake as defined by FDA/AACC International to intermediate markers of effect for CVD and diabetes. A recent review (12) concluded that there was insufficient scientific evidence for claims that whole grain reduces the risk of CVD. The evidence for whole grain consumption and lower risk of diabetes is also only suggestive and inconclusive. However, it is important to bear in mind that many large epidemiological studies of long duration are supportive when it comes to relationships between whole grain intake and a reduced risk of several Western diseases $(1,2)$.

Many components in whole grain, such as certain fibers (e.g. $\beta$-glucan), vitamins (e.g. folate and tocols), minerals (e.g. magnesium), and bioactive components (e.g. phytoestrogens and plant sterols), can act as biomarkers or influence biomarkers for the abovementioned diseases. At present it is not possible to verify the specific components in whole grain responsible for reduced risk of disease. There is most likely a combination of different components providing such an effect. The effects of the individual components are impossible to separate, as they are often correlated in different ways to each other. In addition, the effects of consumption of one type of whole grain do not necessarily reflect the magnitude of benefits for other whole grains due to the diversity of whole grains in terms of macronutrients, micronutrients, and bioactive components.

For soluble fibers, i.e. $\beta$-glucan, in certain whole grain foods of oats and barley, health claims based on effect studies have been allowed by FDA from 2006: 'A soluble fiber from food such as (name of food) as part of a diet low in saturated fat and cholesterol may reduce the risk of heart disease. A serving (name of food) supplies (x) grams of the soluble fiber per day to have this effect' (16). The Swedish Code also includes a claim regarding $\beta$-glucan in oats and barley to blood cholesterol level and risk of CVD (14).

Why is there a need for a definition of whole grain? From the existing literature it is evident that there is no internationally accepted definition of whole grain. Some existing definitions are broad and commodity-based, including grains with similar end uses. These definitions include not only cereals (the grass family), but also pseudocereals and will perhaps be extended to other seeds. Other definitions are narrower and include only the most used and studied cereals in the respective population. It is important that international consensus can be reached on this point.

The chemical composition (macronutrients, micronutrients, and bioactive components) of some of the grains suggested for inclusion in the definition is well known, while others are much less well characterized. It is evident that little is known about the composition and health effects of rarely used cereals, such as teff, Job's tears, and fonio, and to some extent the pseudocereals.

For consumers, health authorities, and the relevant industries, the nutritional aspects are the main concern. It is therefore important that relevant scientific knowledge be made available, regarding both composition and health aspects, before including any grain in a definition. Simply considering the starch content, as has been suggested, would not be sufficient. Scientific documentation of health effects must form the basis for inclusion, and it is better to start with a restricted list of grains (a precautionary principle) and extend this as more knowledge becomes available.

It is also important to restrict grains included in the definition to true cereals belonging to the grass family (Poaceae) with similar botanical and chemical composition. Inclusion of all grains, such as pseudocereals and perhaps pulses and other seeds, due to high starch content and the same end uses and regardless of family membership, could be confusing for all those wishing to use the definition.

\section{Two steps are necessary for definition}

In agreeing a definition of whole grain and whole grain food there are two essential steps: definition of the ingredients of whole grain and definition of whole grain foods. It is important to begin by agreeing a definition of whole grain in terms of the raw dry materials (milled, cracked, crushed, rolled, or flaked). A definition of whole grain foods prepared by thermal, enzymatic, and chemical treatments, such as baking, malting, and fermentation, should then only be agreed once evidence on their composition and health effects becomes available.

However, the definition of whole grain food urgently needs to contain information about the effects of processing. An example of this is $\beta$-glucan from oats and barley, which will generally not have the same nutritional effects in a fermented product such as bread as in the minimally processed grains. This is also true for sensitive vitamins such as folate. This is highly relevant information, which must be considered by the food industry when producing foods with desired health effects.

\section{Conclusions}

We believe that an internationally accepted definition of whole grain is the first essential step in establishing health claims for whole grain and whole grain foods. An exact definition of the raw materials and knowledge about the components providing the health effects would appear to be crucial issues for the authorities when approving health claims.

\section{Conflict of interest and funding}

The authors have not received any funding or benefits from industry to conduct this study. 


\section{References}

1. Slavin J. Why whole grain are protective: biological mechanisms. Proc Nutr Soc 2003; 62: 129-34.

2. Mejborn H, Biltoft-Jensen A, Trolle E, Tetens I. Fuldkorn. Definition og vidensgrundlag for anbefaling af fuldkornsindtag i Danmark [Definition and scientific background for recommendations of wholegrain intake in Denmark] (In Danish). Copenhagen, Denmark: Fodevareinstituttet, DTU; 2008, pp. $1-90$

3. De Moura FF, Lewis KD, Falk MC. Applying the FDA definition of whole grains to the evidence for cardiovascular disease health claims. J Nutr 2009; 139: 2220S-6S

4. Best D. Whole seed - better than whole grain. Cereal Foods Worlds 2009; 54: 226-8.

5. Delcour JA, Hoseney RC. Principles of cereal science and technology, 3rd ed. St. Paul, MN: AACC International; 2010 pp. 1-2.

6. Anon. AACC International defines whole grain; 1999. http:// www.aaccnet.org/definitions/wholegrain.asp [cited 1 February 2010].

7. Anon. Health claims meeting significant scientific agreement (SSA); 2006. http://www.fda.gov/Food/LabelingNutrition/Label Claims [cited 1 February 2010].

8. The American Whole Grain Council; 2004. http://wholegrainscouncil.org/whole-grains-101/definition-of-whole-grains [cited 2 February 2010].

9. Anon. Guidelines for the Scandinavian Keyhole; 2009. http:// www.norden.org/en/nordic-council-of-ministers/council-of-ministers/ council-of-ministers-for-fisheries-and-aquaculture-agriculturefood-and-forestry-mr-fjls/keyhole-nutrition-label. [cited 1 February 2010].

10. FDA. Health claim notification for whole grain foods; 1999. http://www.fda.gov/Food/LabelingNutrition/LabelClaims/FDA
ModernizationActFDAMAClaims/ucm073639.htm [cited 2 February 2010]

11. FDA. Guidance for industry and FDA staff: whole grain labeling statements; 2006. http://www.fda.gov/OHRMS/DOCKETS/98fr/06d-0066-gd10001.pdf [cited 2 February 2010].

12. Life Sciences Research Office. Whole grain intake and cardiovascular disease and whole grain intake and diabetes. A review. Bethesda, MD: Life Sciences Research Office; 2008. http:// www.lsro.org/articles/wholeGrainIntake.html [cited 1 February 2010].

13. Anon. Proposed generic health claim, UK Joint health claims initiative; 2002. http://www.jhci.org.uk/approv/wgrainh.htm [cited 1 February 2010].

14. Anon. The Swedish Code of practice on health claims entitled "Health claims in the labeling and marketing of food products: the food industry's rules"; 2009. http://www.snf.ideon.se/snf/en/ rh/Healthclaims.htm [cited 1 February 2010].

15. Willett WC. Nutritional epidemiology, 2nd ed. New York: Oxford University Press; 1998.

16. FDA. Food labeling. Health claims; soluble dietary fibers from certain foods and coronary heart disease; 2006. http://www.fda.gov/ Food/LabelingNutrition/LabelClaims/HealthClaimsMeetingSignifi cantScientificAgreementSSA/ucm074291.htm [cited 2 February 2010].

*Wenche Frølich

Norwegian School of Hotel Management

University of Stavanger

c/o Lindebergveien 39

NO- 1358 Jar, Norway

Email: wenche.frolich@live.no 